\title{
SARS-CoV-2-Related Acute Respiratory Distress Syndrome Uncovers a Patient with Severe Combined Immunodeficiency Disease
}

\author{
Bandar Al-Saud ${ }^{1,2}$ (1) $\cdot$ Khaled M. Hazzazi $^{1} \cdot$ Reem Mohammed $^{1,2} \cdot$ Alaa Al Najjar $^{3} \cdot$ Tariq Al Hazmi $^{4} \cdot$ Dorota Monies $^{5}$. \\ Fowzan S. Alkuraya ${ }^{2,6}$
}

Received: 28 March 2021 / Accepted: 10 May 2021 / Published online: 25 June 2021

(C) The Author(s), under exclusive licence to Springer Science+Business Media, LLC, part of Springer Nature 2021

Keywords COVID-19 $\cdot$ SCID $\cdot$ Adaptive immunity $\cdot$ JAK3 $\cdot$ Compound heterozygous $\cdot$ T cells $\cdot$ Lymphopenia

To the Editor,

The severe acute respiratory syndrome coronavirus 2 (SARS-CoV-2) causes the coronavirus disease 2019 (COVID-19) infection and has led to a major global pandemic [1]. The role of innate and adaptive immunity in COVID-19 infection remains indeterminate. A recent report attempting to characterize the impact of inborn error of immunity (IEI) showed that the defects in innate immunity, such as mutations in genes involved in type I interferon signaling pathway, have been linked to severe COVID-19 diseases [2]. A global survey of 94 patients with IEI and COVID-19 found that patients with adaptive immunity defects had mild or no symptoms [3]. Data that describe a severe impact of COVID-19 infection in patients with severe combined immunodeficiency (SCID) remain scarce [3-5]. Nevertheless, there is compelling evidence showing

Bandar Al-Saud

balsaud@kfshrc.edu.sa

1 Department of Pediatrics, Division of Allergy \& Immunology, Faisal Specialist Hospital \& Research Center, MBC-58, P.O. Box 3354, Riyadh 11211, King, Saudi Arabia

2 College of Medicine, Alfaisal University, Riyadh, Saudi Arabia

3 Department of Pediatrics, Maternity and Children Hospital, Makkah, Saudi Arabia

4 Department of Pediatrics, Intensive Care Unit, Maternity and Children Hospital, Makkah, Saudi Arabia

5 Department of Clinical Genomics, Center for Genomic Medicine, King Faisal Specialist Hospital and Research Center, Riyadh, Saudi Arabia

6 Department of Translational Genomics, Center for Genomic Medicine, King Faisal Specialist Hospital and Research Center, Riyadh, Saudi Arabia that T-cell counts are reduced significantly, surviving $\mathrm{T}$ cells appear functionally exhausted, and this is correlated with COVID-19 disease severity and poor outcome [5-7]. Here, we report on an infant who developed severe COVID19-related acute respiratory distress syndrome in the context of uncorrected SCID.

\section{Case}

An 8-month-old boy was referred to our clinic to investigate SCID and subsequent possible hematopoietic stem cell transplantation (HSCT). He is a product of full-term, uneventful pregnancy and delivery with a birth weight of $3 \mathrm{~kg}$. He was born to non-consanguineous Saudi parents from two different ethnic backgrounds. The family history was significant because of the early death of two siblings due to severe febrile illnesses, one sister and one brother, at the age of 9 months and 22 months, respectively. This patient was well until the age of 5.5 months, when he had fever, cough, and shortness of breath. These symptoms occurred after contact with the mother who had experienced mild symptoms of COVID-19 infection. His nasopharyngeal aspirate (NPA) sample done on the $21^{\text {st }}$ of September 2020, for SARS-CoV2 RNA-PCR, was positive. The patient was admitted on the $23^{\text {rd }}$ of September 2020 as a case of chest infection. His laboratory findings are shown in Table 1. The patient did not show an increase in the inflammatory markers indicative of multisystem inflammatory syndrome (MIS) (Table 1). The patient initially received cefuroxime, but he deteriorated the next day with an increased work of breathing, subsequently requiring oxygen therapy. A chest $\mathrm{X}$-ray showed bilateral infiltrates, and an acute respiratory distress syndrome (ARDS) was diagnosed (Supplementary Fig. 1a). The patient was transferred to the intensive care 
Table 1 Laboratory results

\begin{tabular}{|c|c|c|c|}
\hline Variable & $\begin{array}{l}\text { At presentation (age } \\
5.5 \text { months) }\end{array}$ & $\begin{array}{l}\text { At day }+100 \text { post-HSCT } \\
\text { (age } 12 \text { months) }\end{array}$ & Reference range \\
\hline WBC $\left(\mathrm{mm}^{3}\right)$ & 8920 & 5190 & $6000-1600$ \\
\hline Neutrophil count $\left(\mathrm{mm}^{3}\right)$ & 4760 & 1620 & $1000-700$ \\
\hline Lymphocyte count $\left(\mathrm{mm}^{3}\right)$ & 2520 & 2330 & $4000-12,000$ \\
\hline Hemoglobin (g/dl) & 12.6 & 113 & $10-14.7$ \\
\hline Platelets $10^{3} / \mathrm{UI}$ & 798 & 520 & $150-450$ \\
\hline PT (s) & 11.5 & - & $11-16$ \\
\hline PTT (s) & 1.1 & - & $23-37$ \\
\hline INR & 33.4 & - & $0.8-1.3$ \\
\hline Fibrinogen $(\mathrm{g} / \mathrm{l})$ & 2.4 & - & $1.75-4.48$ \\
\hline Ferritin $(\mathrm{ng} / \mathrm{ml})$ & 52 & - & $20-250$ \\
\hline LDH (international unit/l) & 831 & - & $100-250$ \\
\hline D-dimer $(\mathrm{mg} / \mathrm{l})$ & 0.51 & - & $<0.5$ \\
\hline C-reactive protein $(\mathrm{CRP})(\mathrm{mg} / \mathrm{dl})$ & 0.2 & - & $0.00-0.5$ \\
\hline $\operatorname{ESR}(\mathrm{mm} / \mathrm{h})$ & 5 & - & $0-15$ \\
\hline \multicolumn{4}{|l|}{ Lymphocyte markers } \\
\hline $\mathrm{CD} 3+\left(\mathrm{mm}^{3}\right)$ & 109 & 1633 & $1400-11,500$ \\
\hline $\mathrm{CD} 4+\left(\mathrm{mm}^{3}\right)$ & 43 & 767 & $1000-7200$ \\
\hline $\mathrm{CD} 8+\left(\mathrm{mm}^{3}\right)$ & 66 & 736 & $200-5400$ \\
\hline $\mathrm{CD} 19+\left(\mathrm{mm}^{3}\right)$ & 2950 & 401 & $130-6300$ \\
\hline $\mathrm{CD} 16+\mathrm{CD} 56+\left(\mathrm{mm}^{3}\right)$ & 2 & 476 & $68-3900$ \\
\hline
\end{tabular}

unit and subsequently intubated to receive high-frequency mechanical ventilation. The patient's treatment was accelerated according to the local health authority COVID-19 protocol. The patient received favipiravir $100 \mathrm{mg}$ bid for 5 days, enoxaparin for 10 days, zinc for 10 days, methylprednisolone $5 \mathrm{mg}$ bid for 7 days, and immunomodulatory dose ( $1 \mathrm{~g} / \mathrm{kg}$ ) of intravenous immune globulin for 2 days. The patient's antibiotic was upgraded empirically to vancomycin and piperacillin/tazobactam. Due to persistent lymphopenia and the significant family history, IEI was suspected. A workup showed severe T-cell and NK-cell lymphopenia, with preserved B cells (Table 1). On day 10 of admission, his NPA-PCR sample was negative for SARS-CoV2. His endotracheal sample grew only Candida albicans at day 12, and he was started on amphotericin $\mathrm{B}$. He also received a 14-day course of therapeutic bactrim empirically for Pneumocystis jirovecii pneumonia (PJP) infection. All his blood cultures were negative. At day 21, the patient was extubated and transferred to regular ward. He was discharged home in good condition on day 31 .

At the time we saw the patient in the clinic, he was doing well and gaining weight. Other than the recent admission with ARDS, the mother denied any recurrent fever, infections, oral thrush, or diarrhea. He was up-to-date with his vaccines including rotavirus vaccine without adverse reactions. His physical examination showed a healthy looking infant with normal growth parameters. His tonsils were not visible, but the rest of his examination was unremarkable. His initial immunological workup at our clinic was consistent with the previous results and suggestive of T-B $+\mathrm{NK}-\mathrm{SCID}$. A chest X-ray was repeated and showed normal lung fields and absent thymic shadow (Supplementary Fig. 1b). His serum SARS-CoV2 IgG was not reactive. Serum PCR results for cytomegalovirus, Epstein-Barr virus, and human immunodeficiency virus were all negative.

In view of the patient's clinical status and urgent need to determine eligibility for HSCT, we enrolled the patient under our institutional review board (IRB)-approved protocol for molecular analysis using Flash WES as described before [8]. Variant classification was per ACMG guidelines. Informed consent was obtained from the patient's family. Two previously reported pathogenic heterozygous variants were identified in JAK3: NM_000215.3:c.678_679del:p. (Cys227Profs*49) and NM_000215.3:c.307C > T::p.(Arg103Cys) [9, 10]. Compound heterozygosity was confirmed by Sanger sequencing of both parents, which showed that the father is heterozygous for NM_000215.3:c.307C>T::p. (Arg103Cys) while the mother is heterozygous for NM_000215.3:c.678_679del:p.(Cys227Profs*49). The patient was admitted at the age of 8 months and underwent HSCT from his HLA full-matched brother without chemotherapy conditioning or graft versus host disease prophylaxis. The total number infused for CD34 was $8 \times 10^{6} / \mathrm{kg}$. The patient tolerated the stem cell infusion and was discharged from the hospital after 6 days. Donor chimerism at 
day 26 after the HSCT showed 82\% T-lymphoid engraftment and $0 \%$ myeloid engraftment. The patient was doing well at the last clinic visit at 100 days post-HSCT without transplant-related complications and achieved 95\% T-lymphocyte cell engraftment, $0 \%$ myeloid cell engraftment, and a normal CD3 count (Table 1).

\section{Discussion}

ARDS due to COVID-19 requiring mechanical ventilation is rare in children. In one cohort, it was reported to be $1.7 \%$ of COVID-19 pediatric patients [1]. Having a coexisting medical condition was found to be a risk factor for mechanical ventilation [1]. ARDS was previously reported in patients with adaptive immunity defect [11], and respiratory insufficiency was reported in $31 \%$ in one survey of IEI patients [3]. To date, six patients with the diagnoses for SCID were reported to have COVID-19 infection [3-5]. One patient was post corrected with gene therapy 19 years ago [3], and the other five patients were pre-HSCT [4, 5]. The only case of uncorrected and genetically confirmed SCID who had COVID-19 infection was recently reported for a male infant with a mutation in $I L 2 R G$ casing X-linked SCID [5]. The patient had mild COVID-19 infection in the form of hepatic transaminitis, with the absence of respiratory symptoms. Similar to our patient, he did not develop MIS due to cytokine storm, which may highlight the protective role for both JAK3 and IL2RG gene defects. Although our patient did not show evidence for MIS based on negative inflammatory markers (ESR, CRP, and ferritin), he developed ARDS which indicates a local inflammation to the lungs. In contrast to the reported X-linked SCID patient [5] who had prolonged viral shedding, our patient tested negative for SARS-CoV19 PCR after 10 days. The mean time for pediatrics viral shedding for COVID-19 is 11 days (range 0-24 days) [12]; however, data are lacking for COVID-19 viral shedding in PID patients. Our patient had no NPA sampling for other common respiratory viruses. However, he developed symptoms only after exposure to his symptomatic mother with COVID19 infection. History of contact to confirmed family member with COVID-19 was found in 77\% of COVID-19 infection in children [1]. Moreover, the patient was symptomatic in the month of September, and most of the common respiratory viruses in Saudi Arabia start in the month of October and peak in November to January [13].

In the context of adaptive immunity, previous report showed that rapid induction of SARS-CoV-2-specific CD4 + T cells during acute COVID-19 infection was associated with mild disease [14]. Moreover, a recent study included 4 SCID patients with COVID-19-related lung involvement who had subsequently died before HSCT [4]. Similar to our case, none of the 4 patients developed MIS.
This may suggest that the T-lymphocyte defect is responsible for uncontrolled infection at the site of viral entrance, giving rise to severe respiratory disease, while the patients are somewhat protected from excessive inflammation in the form of MIS. Nevertheless, more studies are needed to evaluate the role of adaptive immunity and T-cell defects against COVID-19 infection.

Our patient's molecular testing showed a compound heterozygous mutation in JAK3 gene. The largest comprehensive study of Mendelian diseases in a consanguineous population revealed that 730 out of 742 autosomal recessive alleles are homozygous (98.4\%) [8]. It is noteworthy, therefore, that this patient belongs to the $1.6 \%$ of Saudi patients whose autosomal recessive diseases are caused by compound heterozygosity.

In conclusion, this case highlights a possible role of adaptive immunity in controlling severe COVID-19 infection, and it adds to the spectrum of clinical presentation of COVID-19 infection in SCID patients.

Supplementary Information The online version contains supplementary material available at https://doi.org/10.1007/s10875-021-01063-x.

Author Contribution All authors contributed to the study conception and design. Material preparation, data collection, and analysis were performed by Bandar Al-Saud, Khaled M. Hazzazi, and Alaa Al Najjar. The first draft of the manuscript was written by Khaled M. Hazzazi, and all authors commented on previous versions of the manuscript. All authors read and approved the final manuscript.

\section{Declarations}

Ethics Approval and Consent to Participate Informed consent was obtained from the patient family with King Faisal Specialist Hospital and Research Center IRB approval (RAC 2170028).

Written informed consent was obtained from the parents.

Consent for Publication The participant has consented to the submission of the case report to the journal.

Conflict of Interest The authors declare no competing interests.

\section{References}

1. Lu X, Zhang L, Du H, Zhang J, Li YY, Qu J, et al. SARS-CoV-2 infection in children. N Engl J Med. 2020;382(17):1663-5.

2. Zhang Q, Bastard P, Liu Z, Le Pen J, Moncada-Velez M, Chen J, et al. Inborn errors of type I IFN immunity in patients with lifethreatening COVID-19. Science. 2020;370(6515):eabd4570.

3. Meyts I, Bucciol G, Quinti I, Neven B, Fischer A, Seoane E, et al. Coronavirus disease 2019 in patients with inborn errors of immunity: an international study. J Allergy Clin Immunol. 2021;147(2):520-31.

4. Delavari S, Abolhassani H, Abolnezhadian F, Babaha F, Iranparast $\mathrm{S}$, Ahanchian $\mathrm{H}$, et al. Impact of SARS-CoV-2 pandemic on patients with primary immunodeficiency. J Clin Immunol. 2021;41(2):345-55. 
5. van Oers NSC, Hanners NW, Sue PK, Aquino V, Li QZ, Schoggins JW, et al. SARS-CoV-2 infection associated with hepatitis in an infant with X-linked severe combined immunodeficiency. Clin Immunol. 2021;224:108662.

6. Diao B, Wang C, Tan Y, Chen X, Liu Y, Ning L, et al. Reduction and functional exhaustion of $\mathrm{T}$ cells in patients with coronavirus disease 2019 (COVID-19). Front Immunol. 2020;11:827.

7. Lee J, Park SS, Kim TY, Lee DG, Kim DW. Lymphopenia as a biological predictor of outcomes in COVID-19 patients: a nationwide cohort study. Cancers (Basel). 2021;13(3):471.

8. Monies D, Abouelhoda M, Assoum M, Moghrabi N, Rafiullah R, Almontashiri N, et al. Lessons learned from large-scale, first-tier clinical exome sequencing in a highly consanguineous population. Am J Hum Genet. 2019;104(6):1182-201.

9. Mauracher AA, Pagliarulo F, Faes L, Vavassori S, Güngör T, Bachmann LM, et al. Causes of low neonatal T-cell receptor excision circles: a systematic review. J Allergy Clin Immunol Pract. 2017;5(5):1457-1460.e22.

10. Al-Mousa H, Abouelhoda M, Monies DM, Al-Tassan N, AlGhonaium A, Al-Saud B, et al. Unbiased targeted next-generation sequencing molecular approach for primary immunodeficiency diseases. J Allergy Clin Immunol. 2016;137(6):1780-7.
11. Fill L, Hadney L, Graven K, Persaud R, Hostoffer R. The clinical observation of a patient with common variable immunodeficiency diagnosed as having coronavirus disease 2019. Ann Allergy Asthma Immunol. 2020;125(1):112-4.

12. Xu CLH, Raval M, Schnall JA, Kwong JC, Holmes NE. Duration of respiratory and gastrointestinal viral shedding in children with SARS-CoV-2: a systematic review and synthesis of data. Pediatr Infect Dis J. 2020;39(9):e249-56.

13. Farrag MA, Hamed ME, Amer HM, Almajhdi FN. Epidemiology of respiratory viruses in Saudi Arabia: toward a complete picture. Arch Virol. 2019;164:1981-96.

14. Tan AT, Linster M, Tan CW, Le Bert N, Chia WN, Kunasegaran $\mathrm{K}$, et al. Early induction of functional SARS-CoV-2-specific T cells associates with rapid viral clearance and mild disease in COVID-19 patients. Cell Rep. 2021;34(6):108728.

Publisher's Note Springer Nature remains neutral with regard to jurisdictional claims in published maps and institutional affiliations. 